\title{
Bioactive components of pomegranate fruit and their transformation by fermentation processes
}

\author{
Małgorzata Gumienna $^{1} \cdot$ Artur Szwengiel $^{1}$ Barbara Górna ${ }^{1}$
}

Received: 20 July 2015 / Revised: 13 October 2015 / Accepted: 24 October 2015 / Published online: 9 November 2015

(C) The Author(s) 2015. This article is published with open access at Springerlink.com

\begin{abstract}
Pomegranate fruits have attracted huge interest among scientists worldwide, thanks to both their chemical composition and sensory value. Diverse varieties, ranging from sweet to sour, may be used in the formulation of products with specific quality and organoleptic attributes. In relation to fresh fruit, products obtained from pomegranate fruits have very attractive sensory attributes, highly desirable for consumers. The aim of this study was to present the chemical composition and the botanical characteristics of pomegranate, as well as the effect of various technological operations and storage conditions of products on the stability of bioactive compounds contained in pomegranate fruits. The process most extensively described and presented by many researchers is fermentation using lactic acid bacteria, yeasts and filamentous fungi. The applicability of fermentation, based on several products such as wine, probiotic beverages, yoghurts and extracts, was shown to yield health-promoting properties, as it was repeatedly documented by many scientists specialized in different fields of science.
\end{abstract}

Keywords Polyphenolic compounds - Antioxidant activity $\cdot$ Fermentation processes $\cdot$ Health-promoting properties

Małgorzata Gumienna

gumienna@up.poznan.pl

1 Department of Fermentation and Biosynthesis, Faculty of Food Sciences and Nutrition, Poznan University of Life Sciences, Wojska Polskiego 31, 60-624 Poznan, Poland

\section{Introduction}

Current trends have led to the concept of a diet in terms of the amounts of nutrients covering the daily requirements of the organism, but also to its perception as a factor improving human health, thus including also components preventing or reducing the risk of chronic diseases. In view of the new perception of food products by consumers, producers need to develop novel foodstuffs meeting all the requirements of buyers. The increasing consumer awareness of the effect of diet on the incidence of risk factors leading to chronic diseases promotes the development of branches of the food industry involved in the production of functional food. The growing concern of consumers over artificial preservatives is an incentive to design foodstuffs preserved using natural methods, such as fermentation. This process enhances digestibility and bioavailability of nutrients and bioactive components found in the product. Taking into consideration these trends, it is clear that pomegranate fruit is an ideal raw material for the production of functional food meeting the requirements of the present-day consumer. In recent years, pomegranate fruits have been investigated by numerous scientists worldwide, as evidenced by hundreds of publications presenting their chemical composition, potential uses and proven health-promoting effects [1-6]. Not everybody knows that pomegranate fruits are prevalent symbols in culture and religion [2, 7]. Pomegranate fruit, due to its structure, has been a symbol of abundant crops and fertility. In arts and architecture, it has been used to decorate churches and temples, palaces and garments [7].

Its health-promoting properties were appreciated already centuries ago, as evidenced by its application against many ailments in folk medicine of the Near East $[2,6]$. Thus, interest of researchers is caused not only by its high contents of bioactive substances, but also its symbolic value 
and presence in culture. Products from pomegranate exhibit a broad spectrum of activity, improving human health, thanks to high contents of polyphenolics. Thanks to its appearance and taste, it is a fruit increasingly often consumed; however, due to its relatively troublesome opening, consumers frequently prefer to consume new products with its addition. In the countries of its origin, it is one of the oldest and most commonly used fruits. It is eaten as fresh fruit, but also in the form of jams, juices, wines and jellies. Increased interest in pomegranate, which we have been observing recently, has contributed to its increased production and import [8]. As it is grown only in tropical and subtropical climates, it was practically unknown in Eastern European countries (including Poland). At present, it is increasingly popular on the Polish market and may be bought in many shops. However, most Eastern European consumers are not aware of products with health-promoting properties comparable to those of fresh fruit. This may result from the limited knowledge of potential buyers on its beneficial effect on the human organism. The fermentation process provides new potential applications for pomegranate to be used in products with very attractive sensory attributes and health-promoting properties, which might become popular on Eastern European market [9].

\section{Botanical and chemical characteristics of pomegranate}

Pomegranate (Punica granatum L.) belongs to the family Lythraceae. It is an angiosperm, typically a shrub or a small tree with an average height of 3-5 m. Leaves are small and elliptic. The red flowers have from 5 to 7 petals and are bisexual. Fruits are orange-sized berries. Fruits are covered by a thick rind ranging in color from yellow to red [10]. Berries are characterized by the presence of internal thin membranes, suspending and protecting seeds surrounded by juicy sarcotestas (aril). When ripe the fruits burst releasing the seeds. Pomegranate is grown in the subtropical and tropical climates. Although it is found in many locations worldwide, the best fruit comes from regions with very high temperatures and a dry climate, having the most advantageous effect on its ripening $[5,10]$. The edible part of the fruit, the sarcotesta, accounts for $52 \%$ of entire fruit weight, including $78 \%$ juice coming from sarcotestas and $22 \%$ seeds (Fig. 1). Fresh juice contains $85.4 \%$ water and $15.6 \%$ dry substance, composed of sugars, organic acids, pectins, anthocyanins, polyphenols, vitamins and minerals. In turn, seeds are rich sources of lipids, proteins and punicalagin. The rind is a rich source of polyphenols, mainly hydrolyzing tannins and anthocyanins, which are responsible for the bitter taste and its coloring [5, 11-13]. The degree of fruit ripeness is assessed mainly based on its external characteristics, such as rind coloring, which, together with the absence of mechanical damage, are decisive factors for consumers. To verify processing quality of fruit, they are tested for contents of acids and sugars as well as physicochemical properties. In Spain, the most popular processing cultivar is cv. Mollar. It is also characterized by large and sweet fruits of intensely red color and soft seeds. The chemical composition of pomegranate is dependent on the variety, location, climate, the degree of ripeness, method of cultivation and storage, while in the case of pomegranate juices it is also connected with the production method, as juice may be produced only from sarcotestas or from whole fruits $[10,14-16]$. Dry matter content in all varieties is approximately $16 \%$ [13]. Sugars account for the greatest share in dry substance of pomegranate fruits, e.g., cultivar

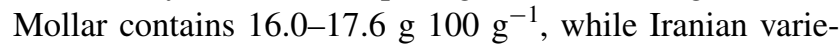

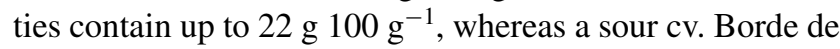

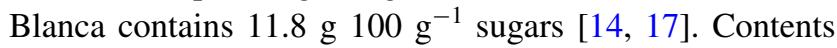
of organic acids also vary, with citric acid predominating. In sweet cultivars, the total content of these compounds is

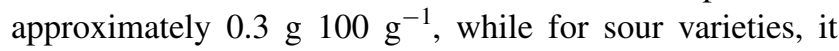

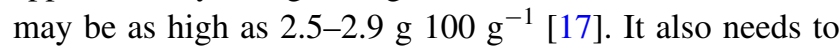
be mentioned here that pomegranate fruit contains ascorbic acid at $15 \mathrm{mg} 100 \mathrm{~g}^{-1}$ (cv. Shirin-e-Bihaste). The contents and variety of antioxidants found in pomegranate fruit are impressive and dependent primarily on the part of fruit [18]. A good example to illustrate this statement may be punicalagin, which is found at high concentrations only in juice pressed from whole fruits, since it is found in the rind [13]. The most common antioxidants include ellagitannins, anthocyanins, gallic and ellagic acids. The total polyphenol content in juice from fresh sarcotestas is $2117 \mathrm{mg} \mathrm{L}^{-1}$, in juice produced on a commercial scale it is $2566 \mathrm{mg} \mathrm{L}^{-1}$, while juice obtained from Chinese sweet varieties contains $1596 \mathrm{mg} \mathrm{L}^{-1}$ total polyphenols $[5,13,18,19]$. The amount of anthocyanins in pomegranate is also a cultivardependent trait, and it also depends on the degree of fruit ripeness. Their content in cv. Mollar de Elche is $141.9 \mathrm{mg}$ $\mathrm{L}^{-1}$ juice, while in cv. Wonderful, it is $306 \mathrm{mg} \mathrm{L}^{-1}$. Pomegranate fruits are also good sources of sodium, potassium [20], iron, zinc, magnesium, copper, calcium, phosphorus and manganese - both in seeds and in juice [16].

\section{Characteristics of bioactive components found in pomegranate fruits}

A bioactive substance is a substance, whose consumption at small amounts has a beneficial effect on human health, extending outside its basic nutritional function and at the same time not being a medicinal substance. Its spectrum and effectiveness may be determined using biomarkers. Incorporation of these substances into our daily 


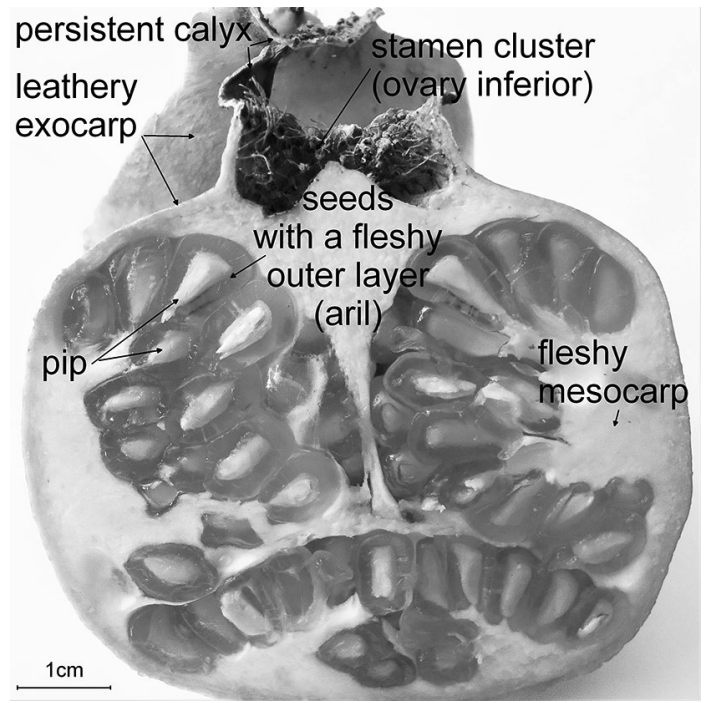

Fig. 1 Pomegranate fruits (Punica granatum L.) — vertical section

diet makes it possible to reduce the risk of chronic diseases and improve the overall physiological conditions of the organism. Antioxidants found in plants are secondary metabolites serving several functions. They are substances attracting insects-anthocyanins, and as components protecting against UV radiation, or factors regulating osmotic pressure, as well as exhibiting an astringent effect [21, 22]. They also exhibit many characteristics having an advantageous effect on human health, e.g., inhibiting oxidation reactions, binding pro-oxidative substances, inhibiting enzymes involved in oxidation reactions or scavenging free radicals. Antioxidants stabilize and protect polyunsaturated fatty acids in food, thanks to reactions with free radicals, chelation of metals and disturbing or interrupting the propagation phase of lipid oxidation. They also influence sensory attributes of fruit-derived products. This is evidenced, e.g., by the tart taste of pomegranate fruits caused by their high contents of polyphenols. Forming complexes with different juice components, they may cause its cloudiness or precipitation of sediment. Most typically they are complexes of polyphenols with proteins [11]. In the last several years, bioactive components contained in pomegranate fruits have been investigated by scientists. These analyses focus mainly on the contents of antioxidants or the positive effect on the human organism [1, 2, 4, 5, 13].

\section{Ellagitannins}

These compounds are hydrolyzing tannins exhibiting high reactivity and biological activity, in the center of their molecule containing a monosaccharide (most often glucose), whose hydroxyl groups are partly or completely esterified by gallic or ellagic acid radicals. Ellagitannins are water soluble and readily hydrolyzed by weak acids and bases and readily undergo enzymatic hydrolysis. They may form complexes with polysaccharides, proteins, as well as metal ions $[22,23]$. Also they tend to form high-molecular dimers and oligomers through a $\mathrm{C}-\mathrm{O}-\mathrm{C}$ bond. The chemical structure of these compounds determines their chemical and physical properties, biological activity and hydrolyzability. Being compounds of a complex structure, they readily undergo chemical reactions, such as transformation or isomerization. They are hydrolyzed in the alimentary tract by intestinal microflora to a sugar molecule and molecules of ellagic and gallic acids [23, 24]. Metabolism of these compounds is initiated by the decomposition of the ellagitannin to sugar and a molecule of ellagic acid, which is further transformed to urolithins in the small and large intestines. Urolithins are absorbed to the portal hepatic circulation, where they may serve the role of hormone analogs. Bioavailability of ellagitannins is dependent on the location, at which these molecules are absorbed, the efficiency of their release from nutrients during digestion, their concentration and interactions with other components found in the medium [24]. It needs to be stressed that absorbability of these compounds in the human organism is limited. They were detected in intestinal epithelial cells, molecules of punicalagin were found in liver and kidney cells, while they were not observed in lungs, the brain or the heart [24]. This may be connected with the formation of insoluble complexes of these particles with magnesium and calcium ions as well as proteins, as a result of which they may not participate in chemical changes or biotransformation in the organism [22-24]. Ellagitannins are investigated in terms of their antibacterial, antitumor and anti-inflammatory properties [24]. Contents of these compounds in pomegranate fruits include $1500-1900 \mathrm{mg} \mathrm{L}^{-1}$ punicalagin (cv. Wonderful), while ellagitannins with ellagic acid are found at $2020-2660 \mathrm{mg} \mathrm{L}^{-1}$ (cv. Wonderful) and $5700 \mathrm{mg} \mathrm{L}^{-1}$ (cv. Mollar) [25].

\section{Ellagic acid}

This representative of phenolic acids is composed of four lipophilic rings, four phenolic groups and two lactones, which may play a role of electron acceptors, as well as exhibit hydrophilic properties. Ellagic acid may be found in pomegranate fruits in a complexed form as ellagitannins, from which it is released under the influence of hydrolysis. It is mainly found in sarcotestas of pomegranate seeds, is water insoluble [22-27]. The acid contents in individual parts of fruit vary; thus, in juice pressed from sarcotestas, it is only $15.3 \mathrm{mg} / \mathrm{L}$, while it is most concentrated in the rind at $637.7 \mathrm{mg} / \mathrm{kg}[4,13]$. This compound is an antioxidant, thanks to its ability to inhibit oxidation reactions and scavenge free radicals. Ellagic acid has found many applications 
in food, pharmaceutical and cosmetics industries, thanks to its biological activity and properties. It is used as a biomarker to estimate bioavailability of ellagitannins in food components, and to standardize rind extracts and also in the pharmaceutical industry as an antihemorrhagic substance. Ellagic acid is not produced on a commercial scale due to high costs of this process. Research is being conducted on its microbiological production using the Aspergillus Niger GH1 strain $[4,26,28]$.

\section{Anthocyanins}

They are flavonoids due to the C6-C3-C6 carbon skeleton in their molecules, derivatives of the flavylium cation found in the oxo or carbonium forms, their huge diversity resulting from the many potential attachment sites for functional-methoxy and hydroxy — groups in the cation ring. In food, they are mainly found as anthocyanidin mono-, di- and triglycosides [29]. They are natural nonnutritive compounds with antioxidant properties. They are responsible for the color of the rind and sarcotestas ranging from orange to purple, depending on ambient $\mathrm{pH}$. In pomegranate fruit cells, they are accumulated in vacuoles and their content in pomegranate is dependent on the variety and the degree of fruit ripeness. Researchers have shown that during fruit ripening contents of individual anthocyanins change, as in the early stages of maturation delphinidin is the dominant form, while upon reaching physiological maturity cyanidin is found at the greatest concentration, which was confirmed by other studies on the chemical composition of fully ripe pomegranate. Next to these two forms, pelargonidin is also found in lesser amounts [5, $9,12,14]$. These forms are mainly present in the form of mono- or diglycosides. Anthocyanins are unstable compounds; their stability is affected by several factors, ranging from access to oxygen, light and ambient $\mathrm{pH}$ to temperature of heat treatment and storage. Under the influence of these factors, they change color in the range from orange to blue, form yellow or colorless chalcones and finally form brown polymers [30].

\section{Stability in technological processes}

In order to facilitate the use of a raw material in a technological process, it is necessary to determine its chemical composition, as well as chemical and physical properties and stability of compounds contained in the material. Without this information, we are unable to run the process in a manner guaranteeing product safety and quality, when speaking of quality consumers increasingly often point to contents of bioactive substances in food products; thus, in the production process, it is essential to retain their possibly greatest amounts in an unchanged form. At present, it is possible to design and produce food products with specific characteristics meeting the requirements of the market and thus also consumer expectations, applying advances of many fields of science, e.g., genetic engineering, chemistry and molecular biology as well as an appropriate course of the technological process.

\section{Stability of juice}

Depending on pomegranate variety and juice production technology, the products differ in their chemical composition and thus organoleptic attributes. Juices obtained from sarcotestas alone contain approximately $15 \%$ solids, and the level is lower than in the case of juices produced from whole fruits, in which it is $16-17 \%$. This is because sarcotestas are mainly sources of sugars, acids and minerals, while the rind is rich in polyphenolic compounds [11, 15]. Juice pressed from whole fruits has a tart and bitter taste due to the high content of phenolics. The high content of anthocyanins in juice from pomegranate fruits is susceptible to changes in color during the technological process and storage caused by numerous factors. Due to the considerable contents of polyphenolic compounds in juice, they have to be subjected to several technological operations improving their sensory value. Drinks made from pomegranate are clarified to prevent clouding. This process may be performed using various methods. The application of pectinolytic enzymes does not provide satisfactory effects, since juice during storage lasting several weeks becomes cloudy, although it ensures the lowest losses of bioactive substances [15]. Physical methods, e.g., the use of membranes, are too expensive to apply in the production of juice. Clarification using filters is cheaper, but does not guarantee good results [11]. Studies conducted by Trufan et al. [31] suggest the application of other clarifying agents apart from gelatin, e.g., bentonite to remove proteins and kieselsol to remove gelatin. Clarification using gelatin yields very good results; however, its dose has to be adjusted to the contents of agents causing juice. Clarification is connected with a considerable reduction in phenolic contents in juice, and thus also its antioxidant activity [11, 31]. Pasteurization aims at the elimination of microflora found in juice, which may result in deterioration of its quality. Microorganisms naturally found in juice are aerobic forms, lactic acid bacteria and yeasts. It was shown that a very short pasteurization at $95{ }^{\circ} \mathrm{C}$ for 30-60 s eliminates all microorganisms found in juice. Rapid pasteurization at high temperature is also preferred due to much lesser anthocyanin degradation, which promotes maintenance of color in pomegranate juice. Despite a reduction in polyphenolic contents, processed juices still exhibit good antioxidant activity. Nonprocessed juices have inferior quality attributes and a very short shelf life $[11,21,31,32]$. 


\section{Stability of ellagitannins}

Since they are hydrolyzing tannins, ellagitannins are hydrolyzed under the influence of weak acids and bases, as well as enzymes. They are stable in neutral solutions. Tannin acyl hydrolase, called tannase, is the enzyme responsible for the breakdown of these compounds, as it catalyzes hydrolysis of ester bonds between sugar and ellagic acid. These high-molecular compounds due to their low tolerance to tannase are susceptible to degradation under the influence of microorganisms such as filamentous fungi Penicillium, Trichoderma and Ascochyta, and bacteria Bacillus pumilis and Klebsiella planticola. The reaction with this enzyme causes disruption of ester bonds; as a result, a sugar molecule is released and it is used as a source of carbon for microorganisms and molecules of hexahydroxydiphenolic acid, which undergoes spontaneous lactonization in the aqueous medium forming ellagic acid. During the digestion process, ellagitannins are hydrolyzed in the stomach under the influence of hydrochloric acid and undergo further transformations in the successive elements of the alimentary tract [24].

\section{Stability of ellagic acid}

Analyses showed that ellagic acid is a substance of high stability when exposed to various factors. It was shown that a 4-month storage of ellagic acid extract from pomegranate rind at access to light caused no changes in color or clarity of the extract. Ellagic acid shows high stability within a wide range of temperatures; its melting point is $350{ }^{\circ} \mathrm{C}[22$, 28]. Similarly, a 4-month storage of the extract at $45{ }^{\circ} \mathrm{C}$ and $75 \%$ humidity caused no changes, and no changes were observed during 4-month storage of this extract at 4 and $30{ }^{\circ} \mathrm{C}$. It may be stated that this compound is stable during long-term storage. Analyses of the effect of ambient $\mathrm{pH}$ on the content of ellagic acid in the extract showed that at various $\mathrm{pH}$ values, the solution varies in color-at $\mathrm{pH} 5.5$ it is yellow, at $\mathrm{pH} 7$ it is brown-yellow, while at $\mathrm{pH}$ 8 it is brown. It was also shown that storage of extracts at these $\mathrm{pH}$ values caused a marked change in the contents of this compound after a period of 4 weeks. Reduction in its concentration may be explained by hydrolysis of the ester group of ellagic acid to hexahydroxydiphenolic acid [28].

\section{Stability of anthocyanins}

Anthocyanins, particularly in combination with glycosides, exhibit low stability in relation to temperature and heating time, access to oxygen and light. In the aqueous medium, they are unstable; however, it needs to be stressed that they are more stable in acid rather than basic environment. They change color under the influence of changing $\mathrm{pH}$ : At acid
$\mathrm{pH}(\mathrm{pH}<3)$ they turn red, at neutral $\mathrm{pH}(\mathrm{pH}=7)$ they are violet, while at basic values $(\mathrm{ph}>11)$ the solution is blue. The number of substituents and their type also affect their stability. A greater number of hydroxyl groups at the B ring cause a change in color from orange through red to violet, while substitution of these groups with methoxy groups results in the transition from violet to orange. Complexing of anthocyanins with metal ions causes similar effects. During short-term heating (for several minutes), even at $100{ }^{\circ} \mathrm{C}$ no considerable changes are observed in their contents or color of solutions [30, 33]. Blanching has a beneficial effect on contents of anthocyanins, since it inactivates enzymes involved in hydrolysis of these compounds [34]. During analyses of sensitivity to light, it was observed that a reduction in anthocyanin contents was lower at no access to light and the best effects were found at the storage of anthocyanin solution at $\mathrm{pH} 3,10{ }^{\circ} \mathrm{C}$ and with no access to light. Aerobic and anaerobic conditions had no significant effect on the reduction in anthocyanin concentrations in samples. The stability of anthocyanins may be enhanced by copigmentation of these compounds with flavones [30, 34]. They are very sensitive to the action of $\beta$-glucosidase, which causes disruption of bonds with sugar radicals, leading to the formation of anthocyanidins, which exhibits a greater capacity to inhibit free radicals. This enzyme is produced by lactic acid bacteria Lactobacillus and yeasts from the genus Saccharomyces [35, 36].

\section{Analysis of the effect of fermentation on bioactive components of pomegranate fruit}

Fermentation is a very effective method of food product biopreservation, as changes taking place in the course of this process result first of all in the extension of shelf life, modifying sensory attributes of the final product, i.e., its taste, aroma and texture. Chemical reactions occurring during this process frequently contribute to improvement of nutritive value by enhancing bioavailability of compounds and improving their digestibility, but they may also contribute to the formation of new compounds exhibiting many beneficial properties for human health. Juice to be fermented is subjected to several preliminary treatments. Operations preceding fermentation include enzymolysis, clarification, filtration and sterilization. Due to high contents of enzymes-polyphenol oxidase and $\beta$-glucosidase, potentially influencing the composition of phenolics, enzymolysis is run in order to inhibit their activity $[16,35,36]$. Clarification and microfiltration aim at the elimination of sediment and clouding, which deteriorate sensory attributes of juice. Juice prior to fermentation is typically sterilized to preserve the greatest possible amounts of anthocyanin pigments. All these operations cause changes in 
juice composition, and thus also in its contents of bioactive substances. Juice from different parts of pomegranate fruits due to high contents of sugars is a good medium for alcoholic and lactic acid fermentation [5, 16, 19, 35, 37]. It was shown that filamentous fungi grow well on substrates containing different parts of pomegranate fruits due to high concentrations of hydrolyzing tannins, water-holding capacity and water activity, a precondition for the development of these organisms on the media [38].

\section{Fermentation using yeasts from the genus Saccharomyces}

Fermentation by yeasts (Saccharomyces ssp.) and aging of wine result in changes of native substances into secondary metabolites, influencing sensory attributes of the product. Changes occurring during fermentation and its effect on bioactive compounds in pomegranate fruits result first of all from interactions of these compounds with yeast cells. Differences in contents of bioactive components before and after fermentation are caused by reactions of polymerization, condensation, oxidation, hydrolysis, enzyme activity and interactions of antioxidants with yeast cell walls. All the experiments showed reduced contents of phenolic compounds, including anthocyanins, following fermentation. Activity of yeasts during fermentation has a significant effect on phenolics, which modify organoleptic properties of wine, such as tart taste, color and aroma [9, 16, 19, 27, 39]. Several mechanisms are responsible for the decreased contents of these compounds in wine made from pomegranate fruits. The mechanism of antioxidant oxidation is caused by the release of free radicals by yeasts. However, mild oxidation of these compounds may contribute to an improvement of color and reduction in the tart taste in the final product. Hydrolysis reactions also influence a decrease in the contents of antioxidants, particularly ellagitannins, which as hydrolyzing tannins contain a molecule of sugar, consumed in the process of fermentation as a source of carbon. During fermentation, the concentration of gallic acid increased significantly, which may be explained by the hydrolysis of ellagitannins, as well as other oxidation reactions. The presence of phenols during wine aging has a marked effect on the release of polysaccharides from cell walls during autolysis of yeast cells, while these in turn contribute to the modification of sensory attributes of pomegranate wines $[9,36]$. Reduced concentration of ellagic acid in wine is explained by researchers by oxidation reactions and precipitation of insoluble sediment. It needs to be stressed that a decrease in the concentration of this compound was greater in the case of cv. Mollar de Elche than in cv. Wonderful; mechanisms of protection or preservation of this component may take place in the latter cultivar. It also needs to be mentioned here that as a result of fermentation, wine with $8.3-9.1 \%$ alcohol by volume is produced, which has a positive effect on solubility of ellagic acid and thus its improved bioavailability. Another cause for changes in the composition of phenolic acids may be connected with their absorption on the surface of yeast cell walls $[9,16]$. Reactions of polyphenol condensation with protein, leading to the formation of sediment, or the condensation of polyphenols with anthocyanins also result in decreased amounts of bioactive components in pomegranate wine [38]. The only bioactive components found at constant concentrations in both juice and wine are flavonoids. Researchers isolated from this group anthocyanins and conducted a separate analysis on the latter. The effect of yeast on anthocyanins determines the color of the final product, which is one of the quality attributes of wine. All studies showed a reduction in anthocyanin contents following fermentation $[9,16,19,27$, 39]. Yeasts may improve wine color, thanks to the formation of weak and reversible bonds between anthocyanins and their cell walls, or through amylolytic enzymes, most typically periplasmic $\beta$-glucosidase, which may cause hydrolysis of anthocyanin molecules to anthocyanidins and glycosides. Another mechanism may be connected with reactions of anthocyanin polymerization with acetaldehyde, generated by the metabolic activity of yeast, with the formation of these complexes causing the formation of yellow pigments. Losses of colored compounds are also generated by their oxidation with elemental oxygen. It also needs to be stressed here that analogously as in the case of contents of antioxidants, an improved color stability was found for $\mathrm{cv}$. Wonderful, which may be caused by their greater concentration $[9,36]$. Experiments conducted on varieties grown in China showed reduction in anthocyanins, in the case of wines produced from sar-

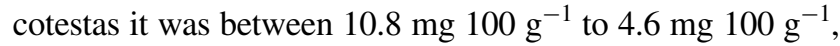
while in wine produced from whole fruits it was $6.7 \mathrm{mg}$ $100 \mathrm{~g}^{-1}$ to $3.3 \mathrm{mg} 100 \mathrm{~g}^{-1}$ [16]. Other analyses showed that sulfitation at $50 \mathrm{mg} \mathrm{SO}_{2} \mathrm{~L}^{-1}$ in sweet and red wines may increase contents of anthocyanins [19]. When describing metabolism of antioxidants, we need to remember about their antioxidant activity. Contents of bioactive substances in pomegranate wines amount to approximately $750 \mathrm{mg}$ $\mathrm{L}^{-1}$-in wines produced from sarcotestas only, while in wines from whole fruits the level of these components was as high as $2500 \mathrm{mg} \mathrm{L}^{-1}[16,19]$. For comparison, red wine contains $2038 \mathrm{mg} \mathrm{L}^{-1}$ and green tea contains $1029 \mathrm{mg} \mathrm{L}^{-1}$, which confirms the thesis that contents of these compounds in pomegranate products are higher than in the most popular drinks with high concentrations of antioxidants [13, 27]. Although contents of phenolic compounds decreased during fermentation, it was still greater in comparison with wines produced from other berry fruits [40]. The capacity to inhibit free radicals is also high, in the case of hydroxyl radicals ranging from 75 to $90 \%$. The antioxidant activity 
in relation to the peroxide radical varies, with the best found for dry wine at approximately $80 \%$, while its relation to the DPPH radical the inhibitory capacity is the lowest, amounting to $15-45 \%$. A certain activity was also observed in relation to the $\mathrm{ATBS}^{+}$radical. Polyphenols from fermented juice inhibit the activity of lipoxygenase, responsible for oxidation of fatty acids and formation of free radicals [9, 19, 27].

\section{Fermentation using lactic acid bacteria}

Lactic acid fermentation is an important biopreservation process, which may enhance or maintain nutritive and sensory value of the product. Bacteria used most frequently by researchers to run lactic acid fermentation include strains of Lactobacillus plantarum, L. acidophilus, L. delbruekii and L. paracasei [35, 37, 41]. Experiments showed that the above-mentioned probiotic strains are capable of surviving in a medium such as juice produced from pomegranate fruits [41]. Each analysis of fermented juice showed reduced contents of bioactive components [35, 37]. Similarly as in the case of alcoholic fermentation, the greatest change in their contents was observed for anthocyanins. It was caused by hydrolysis of anthocyanin molecules by $\beta$-glucosidase to sugar radicals and anthocyanidins. However, those studies also showed a greater capacity to inhibit free radicals in fermented juice in comparison with fresh juice, which is explained by the transformation of anthocyanins to anthocyanidins, exhibiting greater antioxidant activity in relation to DPPH radicals [35]. A study conducted by Filannino et al. [37] showed that reduced contents of bioactive components during fermentation also cause a decrease in antioxidant capacity. However, reduction in this capacity seems to be proportional to the reduction in phenolic concentrations. We also need to stress here that it was nonfermented juice which exhibited the lowest capacity to inhibit free radicals, while fermented juices showed a constant antioxidant activity. The concentration of ellagic acid was also shown to increase. A similar explanation may be given here as for alcoholic fermentation, i.e., hydrolysis of ellagitannins caused a greater acid concentration. Lower concentrations of bioactive components during storage were recorded in nonfermented juice in comparison with fermented juice. Thus, it may be concluded that fermentation, despite reducing the concentration of polyphenols, contributes to their stabilization and reduces losses during storage in the product made from pomegranate fruits. The experiments showed that the color of fermented juice was more stable during storage than that of nonfermented juice [37]. This may indicate that anthocyanins, which were retained in juice, exhibit a lesser tendency to color changes or browning.

\section{Fermentation using filamentous mold Aspergillus niger}

Analysis of the fermentation process using a strain of $A$. niger was run on the rind or pomace formed in the production of juice or another product [26, 38, 42]. The rind was selected as the object of analyses, because it contains the greatest amounts of bioactive compounds, particularly hydrolyzing tannins $[5,38]$. In contrast to seeds, powdered rind is a preferred material used in fermentation on solid medium, thanks to the higher content of polyphenols and a greater water-holding capacity. Water activity, which is a necessary prerequisite for growth of these microorganisms, ranges from 0.88 to 0.99 , and the one recorded for pomegranate rind is aw $=0.98$. The A. niger strain is considered to be a very good producer of tannin alcyl hydrolase, which causes degradation of ellagitannins and release of ellagic acid [38]. All studies showed an increase in the concentration of this component in the medium-from trace amounts before fermentation to the level after fermentation ranging from $6.3 \mathrm{mg} \mathrm{g}^{-1}$ to $8.4 \mathrm{mg} \mathrm{g}^{-1}$ in the rind, while other studies reported an increase in the concentration from 0.2 to $0.9 \%[26,38,42]$. It was shown that ellagic acid produced by the microbiological method exhibited high chemical purity. In their study, Robledo et al. [38] stated that the potential amount of ellagic acid produced by this method is $8 \mathrm{~kg}$, which could be obtained from 1 ton pomace generated in the production of juices from pomegranate fruits. To date, the production of ellagic acid has been considered problematic due to its high costs and low efficiency; however, these analyses may prove useful in the development of a commercial-scale method to generate this bioactive compound.

\section{Potential use of pomegranate fruits to obtain products with health-promoting properties}

An attempt at the analysis of the fermentation process suggests that this process may yield pomegranate products exhibiting properties of functional food. Fermentation provided good effects, generating attractive products. Although contents of bioactive substances are degraded during this process, those retained in the product exhibit good stability during storage and high antioxidant activity. Reduction in anthocyanins to anthocyanidins caused an increase in this activity [9, 41]. Analysis of alcoholic fermentation showed that it is possible to produce attractive wine, having a beneficial effect on the human organism. Wine from pomegranate fruits was characterized by very high capacity to inhibit free radicals, and this proves to be crucial for health-promoting properties. Alcoholic fermentation is also a method to utilize a raw material unattractive for consumers, such as mechanically damaged fruits 
and fruits with uneven rind coloring. Color and the aroma bouquet were attractive for potential consumers. Using different pomegranate varieties, we may produce sweet and dry wines, varying in their contents of polyphenols and alcohol [16, 19]. It was assumed by the researchers that fermentation run using probiotic bacteria should make it possible to produce a novel, nondairy probiotic drink, which could be a very good product for individuals with lactose intolerance. Conducted analyses showed that juice from pomegranate fruits is a good medium for growth of probiotic bacteria, with their count being stable during 2-week storage of fermented juices at $4{ }^{\circ} \mathrm{C}[35,41,43$, 44]. Such a product would also be beneficial for diabetics due to its capacity to enhance the activity of the enzyme responsible for the regulation of blood glucose level. Fermentation using Aspergillus niger strains showed a potential application for pomace of pomegranate fruits generated after juice production as a very good material for the production of pure ellagic acid $[26,36,42]$. Other studies showed potential applicability of sediment formed during pomegranate wine production as a raw material to obtain polyphenols [45]. Excessive concentration of polyphenols, particularly tannins, contributes to the tart and bitter taste of juices or wines, which are not attractive for consumers [11, 15]. Treatments facilitating the generation of polyphenolic compounds from pomace would be applied in the case of these food products to increase their contents of these compounds, thus increasing their antioxidant properties at no deterioration of sensory attributes. They may be supplemented to food products in the form of microcapsules, which exhibit stability during 90-day storage [46]. Potential applications for polyphenolic compounds obtained by fermentation of waste product after juice pressing may include films and edible coatings, as these compounds thanks to their properties may constitute a barrier against growth of microflora on the surface of fruits and vegetables [47]. A good product with promising prospects for success on the European market is yoghurt with an addition of pomegranate juice $(40 \%)$. This product during analyses had a good composition of bioactive compounds and antioxidant activity. It exhibited good stability during storage, although it was lower than that of fresh juice [48]. It is clear that pomegranate fruits are rich sources of bioactive substances and may be used to obtain many products, including those which may be considered functional food. Fermentation facilitates generation of novel products with good sensory attributes and at the same time exhibiting good stability. It may be a management method for waste (pomace generated by juice production) to obtain bioactive substances, which may be used in supplementation of food products.

\section{Concluding remarks}

It is clear that pomegranate fruits are rich sources of bioactive components and minerals. Although pomegranate grows in tropical and subtropical climates, which prevents its cultivation in most European countries, its availability is constantly increasing. Research on this fruit is being conducted only in the countries where it is grown and for centuries present in their culture and local cuisine. For these reasons, knowledge on its health-promoting properties and chemical composition is not widespread in Europe. The spectrum of its beneficial effects on the human organism is extensive and suggests that it would be advisable to introduce it in everyday diet. It is a good product for diabetics, patients suffering from coronary heart disease or even cancer. Both pomegranate fruits and products obtained from them could be used as preventive measures to reduce the risk of incidence of certain diseases.

Fermentation using yeasts and lactic acid bacteria changes the chemical composition of juices, contributing to modification of their properties. As it was stated before, the primary bioactive substances found in pomegranate fruits, i.e., ellagitannins and anthocyanins, are not stable during juice processing, hindering development of novel products, which could be launched on the market as health-promoting products. Although fermentation reduces the contents of bioactive components, at the same time it improves the stability of both those which were transformed and those which remained unchanged. This fact suggests that this process has an advantageous effect on the maintenance of health effects of these antioxidants, which were retained in the product. What is more, fermentation modifies sensory attributes of the products and thus contributes to increased consumer desirability of the products. Additionally, transformations of ellagitannins during this process promote an increase in the contents of ellagic acid. New methods to obtain ellagic acid from pomace using filamentous fungi make it possible to produce and apply it in food and pharmaceutical industries.

So it is absolutely worth, especially for European consumers, approaching the pomegranate as a raw fruit as well as its processed products (juices, jams, snacks), presenting the wide range of its beneficial effects on the human health and emphasizing the abundance of biological active compounds with their health-promoting properties.

\section{Compliance with ethical standards}

Conflict of interest None.

Compliance with ethics requirements This article does not contain any studies with human or animal subjects.

Open Access This article is distributed under the terms of the Creative Commons Attribution 4.0 International License (http://creativecommons.org/licenses/by/4.0/), which permits unrestricted use, distribution, 
and reproduction in any medium, provided you give appropriate credit to the original author(s) and the source, provide a link to the Creative Commons license, and indicate if changes were made.

\section{References}

1. Bhandari PR (2012) Pomegranate (Punica granatum L). Ancient seeds for modern cure? Review of potential therapeutic applications. Int J Nutr Pharmacol Neurol Dis 2(3):171-184

2. Jurenka JS (2008) Therapeutic applications of pomegranate (Punica granatum L.): a review. Altern Med Rev 13(2):128-144

3. Mena P, Calani L, Dall'Asta C, Galaverna G, García-Viguera C, Bruni R, Crozier A, Del Rio D (2012) Rapid and comprehensive evaluation of (poly) phenolic compounds in pomegranate (Punica granatum L.) juice by UHPLC-MSn. Molecules 17:14821-14840

4. Viladomiu M, Hontecillas R, Lu P, Basseganya-Riera J (2013) Preventive and prophylactic mechanisms of action of pomegranate bioactive compounds. Evid-Based Complement Alternat Med 2013:1-18

5. Fischer UA, Carle R, Kammerer DR (2011) Identification and qualification of phenolic compounds from pomegranate (Punica granatum L.) peel, mesocarp, aril and differently produced juices by HPLC-DAD-ESI/MS. Food Chem 127:807-821

6. Langley P (2000) Why pomegranate? BMJ 321:1153-1154

7. Engels G, Brinckmann J (2013) Pomegranate. Am Bot Counc 100:1-7

8. Akbarpour V, Hemmati K, Sharifani M (2009) Physical and chemical properties of pomegranate (Punica granatum L.) fruit in maturation stage. American-Eurasian J Agric. Environ Sci 6(4):411-416

9. Mena P, Gironés-Vilaplana A, Martí N, García-Viguera C (2012) Pomegranate varietal wines: phytochemical composition and quality parameters. Food Chem 133:108-115

10. Holland D, Hatib K, Bar-Ya'akov I (2009) Pomegranate: botany, horticulture, breeding. Horticu Rev 35:127-191

11. Oziyci HR, Karhan M, Tetik N, Turhan I (2013) Effects of processing method and storage temperature on clear pomegranate juice turbidity and color. J Food Process Pres 37(5):899-906

12. El-Nemr SE, Ismail IA, Ragab M (1990) Chemical composition of juice and seeds of pomegranate juice. Nahrung 34(7):601-606

13. Gil MJ, Tomás-Barberán FA, Hees-Pierce B, Holcroft DM, Kader AA (2000) Antioxidant activity of pomegranate juice and its relationship with phenolic composition and processing. $\mathrm{J}$ Agric Food Chem 48(10):4581-4589

14. Legua P, Malgarejo P, Martínez JJ, Martínez R, Henrnández F (2012) Evaluation of spanish pomegranate juices: organic acids, sugars, and anthocyanins. Int J Food Prop 15(3):481-494

15. Rinaldi M, Caligiani A, Borgese R, Palla G, Barbanti D, Massini $R$ (2013) The effect of fruit processing and enzymatic treatments on pomegranate juice composition, antioxidant activity and polyphenols content. Food Sci Technol 53:355-359

16. Wasila H, Li X, Liu L, Ahmad I, Ahmad S (2013) Peel effects of phenolic composition, antioxidant activity, and ma king pomegranate juice and wine. J Food Sci 78(8):1166-1172

17. Melgarejo P, Salazar DM, Atrés F (2000) Organic acids and sugars composition of harvested pomegranate fruits. Eur Food Res Technol 211:185-190

18. Sentandreu E, Cerdán-Calero M, Sendra JM (2013) Phenolic profile characterization of pomegranate (Punica granatum) juice by high-performance liquid chromatography with diode array detection coupled to an electrospray ion trap mass analyzer. $\mathrm{J}$ Food Comp Anal 30:32-40
19. Zhuang H, Du J, Wang Y (2011) Antioxidant capacity changes of 3 cultivar Chinese pomegranate (Punica granatum L.) juices and corresponding wines. J Food Sci 76(4):606-611

20. El Kar C, Ferchichi A, Attia F, Bouajila J (2011) Pomegranate (Punica granatum) juices: chemical composition, micronutrient cations, and antioxidant capacity. J Food Sci 76(6):795-800

21. Zarei M, Azizi M, Bashiri-Sadr Z (2010) Studies on physicochemical properties and bioactive compounds of six pomegranate cultivars grown in Iran. J Food Technol 8(3):112-117

22. Sepúlveda L, Ascacio A, Rodriguez-Herrera R, Aguilera-Carbó A, Aguilar CN (2011) Ellagic acid: biological properties and biotechnological development for production processes. African $\mathbf{J}$ Biotechnol 10(22):4518-4523

23. Sibel U, Jale A (2012) Hydrolytic properties of ellagic acid in commercial pomegranate juices. World Acad Sci Eng Technol 6(7):717-720

24. Lipińska L, Klewicka E, Sójka M (2014) Structure, occurrence and biological activity of ellagitannins: a general review. Acta Sci Pol Technol Aliment 13(3):289-299

25. Larrosa M, García-Conesa MT, Espín JC, Tomás-Barberán FA (2010) Ellagitannins, ellagic acid and vascular health. Mol Aspects Med 31(6):513-539

26. Sepúlveda L, Buenrostro-Figueroa JJ, Ascacio-Valdés JA, Aguilera-Carbó A, Rodriguez-Herrera R, Contreras-Esquivel JC, Aguilar CN (2014) Submerged culture for production of ellagic acid from pomegranate husk by Aspergillus niger GH1. Micol Aplicada Int 26(2):27-35

27. Schubert SY, Lansky EP, Neeman I (1999) Antioxidant and eicosanoid enzyme inhibition properties of pomegranate seed oil and fermented juice flavonoids. J Ethnopharmacol 66:11-17

28. Panichayupakaranant P, Itsuriya A, Sirikatitham A (2010) Preparation method and stability of ellagic acid-rich pomegranate fruit peel extract. Pharm Biol 48(2):201-205

29. Joshi Y, Goyal B (2011) Anthocyanins: a lead for anticancer drugs. Int J Res Farm Chem 1(4):1119-1126

30. Oszmiański J (2002) Stabilization and application of anthocyanin chokeberry dye to colouring of beverages. Acta Sci Pol Technol Aliment 1(1):37-45

31. Trufan Ö, Türkyılmaz M, Yemiş O, Özkan M (2011) Anthocyanin and colour changes during processing of pomegranate (Punica granatum L, cv. Hicaznar) juice from sacs and whole fruit. Food Chem 129:1644-1651

32. Mena P, Vegara S, Martí N, García-Viguera C, Saura D, Valero M (2013) Changes on indigenous micro biota, colour, bioactive compounds and antioxidant activity of pasteurised pomegranate juice. Food Chem 141:2122-2129

33. Pojer E, Mattivi F, Johnson D, Stockley CS (2013) The case for anthocyanin consumption to promote human health: a review. Compr Rev Food Sci Food Saf. 12(5):483-508

34. Sadilova E, Stintzing FC, Carle R (2006) Thermal degradation of acylated and nonacylated anthocyanins. J Food Sci 71(8):504-512

35. Mousavi ZE, Mousavi SM, Razavi SH, Hadinejad M, EmamDjomeh Z, Mirzapour M (2013) Effect of fermentation of pomegranate juice by Lactobacillus plantarum and Lactobacillus acidophilus on the antioxidant activity and metabolism of sugars, organic acids and phenolic compounds. Food Biotechnol 27:1-13

36. Salmon JM (2006) Interactions between yeast, oxygen and polyphenols during alcoholic fermentation: practical implications. LWT_-food. Sci Technol 39:959-965

37. Filannino P, Azzi L, Cavoski I, Vincentini O, Rizzello CG, Gobbetti M, Di CR (2013) Exploitation of the health-promoting and sensory properties of organic pomegranate (Punica granatum L.) juice through lactic acid fermentation. Int. J Microbiol 163(2-3):184-192 
38. Robledo A, Aguilera-Carbó A, Rodriguez R, Martinez JL, Garza Y, Aguilar CN (2008) Ellagic acid production by Aspergillus niger in solid state fermentation of pomegranate residues. J Ind Microbiol Biotechnol 35:507-513

39. Ordoudi SA, Mantzouridou F, Daftsiou E, Malo C, Hatzdimitriou E, Nenadis N, Tsimidou MZ (2014) Pomegranate juice functional constituents after alcoholic and acetic acid fermentation. J Funct Foods 8:161-168

40. Heinonen IM, Lethonen PJ, Hopia AI (1988) Antioxidant activity of berry and fruit wines and liquors. J Agric Food Chem 46(1):25-31

41. Mousavi ZE, Mousavi SM, Razavi SH, Emam-Djomeh Z, Kiani $\mathrm{H}$ (2011) Fermentation of pomegranate juice by probiotic lactic acid bacteria. World J Microbiol Biotechnol 27(1):123-128

42. Aguilar CN, Aguilera-Carbo A, Robelo A, Ventura J, Belmares R, Rodriguez- Herrera R, Contreras J (2008) Production of antioxidant nutraceuticals by solid state cultures of pomegranate (Punica granatum) peel and creosote bush (Larrea tridentata) leaves. Food Technol Biotechnol 46(2):218-222

43. Bialonska D, Ramnani P, Kasimsetty SG, Muntha KR, Gibson GR, Ferreira D (2010) The influence of pomegranate by-product and punicalagins on selected groups on human intestinal microbiota. Int J Food Microbiol 140:175-182
44. Plessas S, Koulis M, Alexopoulos A, Bezirtzoglou E (2011) Functional drink production through pomegranate fermentation. 11th international congress on engineering and food (ICEF11) Athens, Greece p. 2029-2030

45. Mena P, Ascacio-Valdés JA, Gironés-Vilaplana A, Del Rio D, Moreno DA, García-Viguera C (2014) Assessment of pomegranate wine less as a valuable source for the recovery of (poly)phenolic compounds. Food Chem 145:327334

46. Çam M, İçyer NC, Erdoğan F (2014) Pomegranate peel phenolics: microencapsulation, storage stability and potential ingredient for functional food development. Food Sci Technol $55: 117-123$

47. Martínez-Avíla GCG, Aguílera AF, Saucedo S, Rojas R, Rodríguez R, Aguílar CN (2014) Fruit wastes fermentation for phenolic antioxidants production and their application in manufacture of edible coatings and films. Crit Rev Food Sci Nutr 54:303-311

48. Trigueros L, Wojdyło A, Sendra E (2014) Antioxidant activity and protein-polyphenol interactions in a pomegranate (Punica granatum L.) yogurt. J Agric Food Chem 62:6417-6425 